\title{
Therapist-in-the-Loop Robotics-Assisted Mirror Rehabilitation Therapy: An Assist-as-Needed Framework*
}

\author{
Mahya Shahbazi, S. Farokh Atashzar, Mahdi Tavakoli, Rajni V. Patel
}

\begin{abstract}
This paper presents a Therapist-in-the-Loop (TIL) framework for robotics-assisted mirror rehabilitation therapy integrated with adaptive Assist-as-Needed (ANN) training, to be adjusted based on the impairment and disability level of the patient's affected limb. Closed-loop system stability has been investigated using a combination of the Circle Criterion and the Small-Gain Theorem to account both for time-delay and the time-varying adaptive ANN training. Experiments to investigate the performance of the proposed framework are reported.
\end{abstract}

\section{INTRODUCTION}

Robotics-assisted bilateral mirror therapy has received a great deal of attention in the past decade [1], [2]. During this type of therapy, motions of the Patient's Functional Limb (PFL) are mirrored through a telerobotic medium to the Patient's Impaired Limb (PIL). Through mirror-symmetric bilateral movements of the two limbs, the unimpaired hemisphere of the brain interacts with the impaired hemisphere, thereby inducing reorganization of the motor cortex networks and facilitating cortical neural plasticity [3], [4]. The effectiveness of mirror-symmetric bilateral therapy has been shown over traditional unilateral therapy, in terms of an increase in the functional ability in addition to a decrease in movement times for the impaired limb [5].

Currently, existing robotics-assisted mirror therapy systems, such as MIME [6] provide a Single-Master/SingleSlave (SM/SS) framework in order for the impaired limb to move according to the mirror-image motions of the functional limb. Due to the restrictive SM/SS architecture, the patient's impaired limb interacting with the slave robot can only receive commands from the patient's functional limb interacting with the master robot. Consequently, a therapist cannot be directly involved in the rehabilitation loop to apply corrective movements, or to monitor/assess the PIL performance through haptics feedback. To address these issues, a Therapist-in-the-Loop (TIL) framework is

\footnotetext{
* This research was supported by the Natural Sciences and Engineering Research Council (NSERC) of Canada under a Collaborative Research and Development Grant \# CRDPJ 411603-10, a Canadian Institutes of Health Research (CIHR) and NSERC Collaborative Health Research Projects (CHRP) Grant \#316170; the Canada Foundation for Innovation (CFI) under grant LOF 28241, the Alberta Innovation and Advanced Education Ministry under Small Equipment Grant RCP-12-021, and Quanser Inc.

M. Shahbazi and S.F. Atashzar are with Canadian Surgical Technologies and Advanced Robotics (CSTAR), and with the Department of Electrical and Computer Engineering, Western University, London, ON, Canada. They are also currently visiting research scholars at the University of Alberta, Edmonton, AB, Canada (email: mshahba2@uwo.ca, satashza@uwo.ca). M. Tavakoli is with the Department of Electrical and Computer Engineering, University of Alberta, Edmonton, AB, Canada (email: mahdi.tavakoli@ualberta.ca). R.V. Patel is with CSTAR, the Department of Electrical and Computer Engineering and the Department of Surgery, Western University (email: rvpatel@uwo.ca).
}

proposed for mirror therapy based on a supervised tri-lateral telerobotic system. Fig. 1 illustrates the overall scheme of the proposed framework. Using the proposed framework, a mirroring behaviour is stablished between the patient's two limbs while desired trajectories are generated by a therapist supervising the loop, which is expected to enhance the treatment by engaging the therapist. The framework is designed such that the PFL acts as a medium between the therapist and the PIL, conditioning the desired trajectories before passing them on to the PIL. Benefiting from the patient's proprioceptive knowledge and self-awareness of workspace limitations, the proposed PFL-mediated approach enables the patient to modify the trajectories desired by the therapist for the PIL in order to avoid painful/uncomfortable maneuvers, of which the therapist may not be aware. Based on the trajectories followed by the PIL, which might have been modified by the PFL, the system provides the therapist with haptic feedback, allowing the therapist to better decide on the level of therapy administered to the patient.

The framework also provide adaptive Assist-as-Needed (ANN) therapy to the patient through a time-varying Guidance Virtual Fixture (GVF). The stiffness of the GVF is proposed to be adaptively adjusted according to the patient's impairment/performance level shown during the therapy. In order to investigate stability of the closed-loop telerobotic framework, a combination of the Circle Criterion and the Small-Gain Theorem is applied, and a set of sufficient stability condition is derived. The proposed stability analysis also addresses instabilities caused by communication time delays between the therapist and the patient, which facilitates the case of tele-rehabilitation, a quite recent development in the rehabilitation engineering field [7],[8].

\section{THE PROPOSED FRAMEWORK}

\section{A. Architecture for the PIL/robot interaction}

In order for the PIL to undergo the mirror therapy, its desired position $x_{d e s, P I L}$ is defined to be the mirror image of PFL's position, $x_{P F L}$, as follows:

$$
x_{\text {des,PIL }}(t)=\beta \cdot x_{P F L}(t)
$$

where $\beta=\operatorname{diag}\left(\beta_{1}, \ldots, \beta_{n}\right)$ is the mirroring matrix, accommodating for the mirroring effect between the functional and the impaired limb along the Sagittal axis; the subscript $n$ refers to the number of Degrees of Freedom (DOF). Depending on the mirroring axis, $\beta_{i}(i=1,2, \cdots, n)$, which is the mirroring coefficient for the $i^{t h}$ DOF, can be +1 or -1 . For example, for mirroring along the $\mathrm{x}$-axis, $\beta_{1}$ will be set to -1 , while 


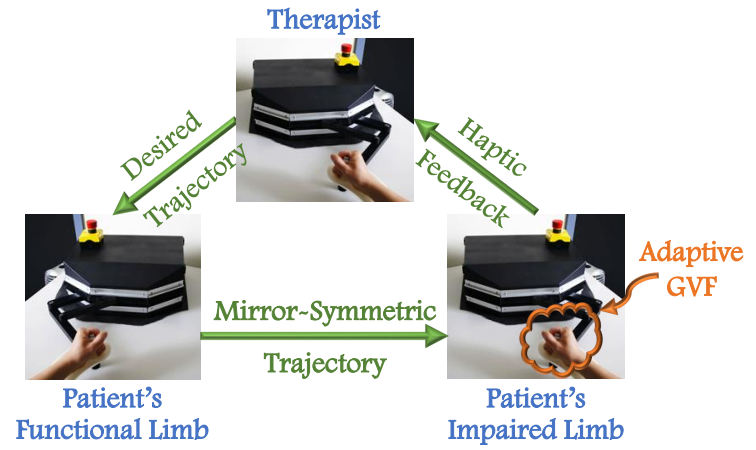

Fig. 1. The overall scheme of the proposed trilateral telerobotic framework $\beta_{i}(i \neq 1)$ will be set to +1 to accommodate for the samedirectional trajectories along other axes.

In order to provide the PIL with an Assist-as-Needed (ANN) therapy, an adaptive GVF is proposed, the stiffness of which can be adaptively adjusted according to the impairment/disability level of the PIL. The higher level of impairment the PIL shows, the more strict and enforcing the GVF gets in order to assist the PIL more. The GVF is designed such that if the PIL remains within a specific range of its desired trajectory, i.e., inside a specific spherical volume centered at the desired trajectory point $x_{d e s, P I L}$, no GVF force will be applied to it. However, if the position error between the PIL and the mirror image of the PFL (the desired trajectory for PIL) exceeds a certain threshold, the GVF will apply force to the PIL in order to assist with accomplishing the trajectory. The allowable range of the position error is set to be up to $R_{G V F}$. Exceeding the allowable range of error, i.e., $\left|x_{\text {des,PIL }}-x_{P I L}\right|>R_{G V F}$, will cause the PIL to receive the following GVF force:

$$
F_{G V F, P I L}(t)=K_{G V F, P I L}(t)\left(x_{d e s, P I L}(t)-x_{P I L}(t)\right)
$$

where $K_{G V F, P I L}(t) \in\left[\kappa_{\min }, \kappa_{\max }\right]$ refers to the adaptive stiffness of the GVF, to be adjusted according to the impairment level of the PIL. $\kappa_{\min }$ and $\kappa_{\max }$ indicate some positive lower and upper bounds to be considered in the design procedure of $K_{G V F, P I L}$. It should be noted that various motor-function assessment metrics, including but not limited to movement accuracy, motion smoothness, movement velocity and grip strength, can be used in order to design the variation profile of the adaptive GVF's stiffness. However, the design of this parameter constitutes our ongoing efforts and will be addressed in the future work. We will allow GVF to be any time-varying virtual fixture in this paper.

In order for the patient to transparently feel the desired GFV force applied by the robot on his/her PIL, it is required to have $F_{P I L}(t)=-F_{G V F, P I L}(t)$, where $F_{P I L}$ indicates the force applied by the PIL to its corresponding robot. Note that the minus sign is to account for the direction of forces, i.e., applied by the robot to the PIL or vice versa.

However, as will be discussed in Section III, similar to any other telerobotic system [9], ensuring closed-loop stability may degrade the system performance and transparency. Thus, to ensure closed-loop stability in the presence of communication delay, a modified impedance surface is defined as the desired closed-loop system at the PIL robot, through which the GVF force $F_{G V F, P I L}$ is applied by the robot to the PIL:

$$
\begin{gathered}
F_{P I L}(t)=-F_{G V F, P I L}(t)+ \\
M_{\vartheta, P I L} \cdot \ddot{x}_{P I L}(t)+B_{\vartheta, P I L} \cdot \dot{x}_{P I L}(t)+K_{\vartheta, P I L} \cdot x_{P I L}(t)
\end{gathered}
$$

where $M_{\vartheta, P I L}, B_{\vartheta, P I L}$ and $K_{\vartheta, P I L}$ stand for mass, damping and stiffness to be used as the local-control parameters at the PIL robot. From the performance perspective, the control parameters are desired to be set to zero, which results in $F_{P I L}(t)=-F_{G V F, P I L}(t)$. However, it will be shown in Section III how positive values for these parameters will contribute to stability of the closed-loop system in the presence of communication time delay between the therapist and the patient in order to facilitate the case of tele-rehabilitation.

\section{B. Architecture for the PFL/robot interaction}

The architecture at the PFL robot is designed such that the PFL receives commands (desired trajectories) from the therapist, but is able to deviate from them. This allows the patient to alter the therapist-commanded trajectory, if the trajectories are felt to be painful or uncomfortable for the PIL. To realize this goal, a position-error impedance surface is designed for the PFL:

$$
\begin{gathered}
F_{P F L, d e s}(t)=M_{\text {des, } P F L}\left(\ddot{x}_{T}^{*}(t)-\ddot{x}_{P F L}(t)\right)+ \\
B_{d e s, P F L}\left(\dot{x}_{T}^{*}(t)-\dot{x}_{P F L}(t)\right)+K_{d e s, P F L}\left(x_{T}^{*}(t)-x_{P F L}(t)\right)
\end{gathered}
$$

where $x_{P F L}$ indicates the trajectory generated by the PFL and $x_{T}^{*}$ refers to the mirror image of the trajectory made by the therapist. Note that since the PIL will move based on the mirror-image of the PFL, while the therapist will provide the trajectory desired for the PIL, the PFL should receive the mirror image of the trajectory commanded for the PIL by the therapist, i.e., to receive $x_{T}^{*}=\beta \cdot x_{T}$, where $\beta$ refers to the mirroring matrix. $M_{d e s, P F L}, B_{d e s, P F L}$ and $K_{d e s, P F L}$ refer to the desired mass, damping and stiffness, through which the PFL can alter the desired trajectories received from the therapist in the interest of safety. Moreover, $F_{P F L, d e s}$ stands for the desired force applied by the robot to the PFL as the result of interaction with the therapist. In order for the PFL to receive $F_{P F L, d e s}$, it is desired to have $F_{P F L}=-F_{P F L \text {,des }}$, where $F_{P F L}$ indicates the force applied by the PFL to the robot. Consequently, and based on the desired impedance surface defined in (4), the position of the functional limb will be:

$$
X_{P F L}(s)=\frac{F_{P F L}(s)}{Z_{d e s, P F L}(s)}+\beta \cdot X_{T}(s)
$$

where $Z_{d e s, P F L}(s)=M_{d e s, P F L} s^{2}+B_{d e s, P F L} s+K_{d e s, P F L}$. Here, $s$ indicates the Laplace operator. Thus, the PFL can follow the therapist's mirrored trajectories $\beta x_{T}$ by applying minimal $F_{P F L}$. However, if the patient decides the trajectories set by the therapist to be painful or uncomfortable for PIL, he or she can apply enough force $F_{P F L}$, des, to alter $x_{P F L}$ from the therapist mirrored trajectory $\beta x_{T}$. The PFL as a medium to convey desired trajectories from the therapist to the PIL increases the safety and comfort for the patient.

With the same reasoning as for (3), for the sake of closed-loop stability, the desired behavior $F_{P F L}=-F_{P F L, d e s}$ 
is replaced by an impedance surface as the desired closedloop system at the PFL robot, through which the desired force $F_{P F L, d e s}$ is applied to the PFL by some modification:

$$
\begin{gathered}
F_{P F L}=-F_{P F L, d e s}(t)+ \\
M_{\vartheta, P F L} \cdot \ddot{x}_{T}(t)+B_{\vartheta, P F L} \cdot \dot{x}_{T}(t)+K_{\vartheta, P F L} \cdot x_{T}(t)
\end{gathered}
$$

where $M_{\vartheta, P F L}, B_{\vartheta, P F L}$ and $K_{\vartheta, P F L}$ refer to the mass, damping and stiffness to be used as the local control parameters at the PFL robot. The control parameters are desired to be zero for the purpose of performance, i.e., the PFL feels $F_{P F L \text {,des, }}$, entirely. However, setting them to non-zero will help with stabilizing the entire closed-loop system.

\section{Architecture for the therapist/robot interaction}

As described earlier, for the purpose of safety and comfort, the framework enables the PFL to alter the therapistcommanded trajectory, $x_{T}$, when necessary. Accordingly, the trajectories followed by the PIL may not be exactly similar to those created by the therapist. Therefore, it is required for the therapist to receive haptic feedback about the PIL movements in relation to the therapist-commanded movements. For this purpose, position-error-based haptic feedback, $F_{\varphi, T}$, is designed to apply to the therapist by his/her corresponding robot, as follows:

$$
\begin{gathered}
F_{\varphi, T}(t)=M_{\varphi, T}\left(\ddot{x}_{P I L}(t)-\ddot{x}_{T}(t)\right)+ \\
B_{\varphi, T}\left(\dot{x}_{P I L}(t)-\dot{x}_{T}(t)\right)+K_{\varphi, T}\left(x_{P I L}(t)-x_{T}(t)\right)
\end{gathered}
$$

where $M_{\varphi, T}, B_{\varphi, T}$ and $K_{\varphi, T}$ denote the mass, damping and stiffness of the position-error-based haptic feedback, respectively. With the same reasoning for (3) and (6), an impedance surface is defined for the desired closed-loop behavior at the therapist side, through which the haptic force feedback $F_{\varphi, T}$ is applied by the robot to the therapist by the modification:

$$
\begin{gathered}
F_{T}=-F_{\varphi, T}(t)+ \\
M_{\vartheta, T} \cdot \ddot{x}_{T}(t)+B_{\vartheta, T} \cdot \dot{x}_{T}(t)+K_{\vartheta, T} \cdot x_{T}(t)
\end{gathered}
$$

where $M_{\vartheta, T}, B_{\vartheta, T}$ and $K_{\vartheta, T}$ stand for the desired mass, damping and stiffness to be used as the local control parameters at the therapist robot. In addition, $F_{T}$ refers to the force applied to the robot by the therapist.

The force $F_{T}$ applied by the therapist to the corresponding robot, as well as the forces $F_{P I L}$ and $F_{P F L}$ applied by the PIL and PFL to their corresponding robots can be modeled by second-order LTI systems [10]:

$$
\begin{aligned}
F_{\Theta}(t)= & F_{\Theta}^{*}(t)-M_{\Theta} \cdot \ddot{x}_{\Theta}(t)-B_{\Theta} \cdot \dot{x}_{\Theta}(t) \\
& \left.-K_{\Theta} \cdot\left(x_{\Theta}(t)-x_{\Theta_{0}}\right)\right)
\end{aligned}
$$

where $F_{\Theta}^{*}$, for $\Theta=P I L, P F L, T$, denote the exogenous force applied by the operator, which is either the patient or the therapist. $M_{\Theta}, B_{\Theta}$ and $K_{\Theta}$ stand for mass, damping and stiffness of the limb, respectively; and $x_{\Theta_{0}}$ indicates the initial position of the therapist's limb, $x_{\Theta}$.

\section{Closed-Loop Stability AnAlysis}

In order to satisfy the desired closed-loop systems locally defined for each robot, (3), (6) and (8), a decentralized impedance controller previously developed by the authors
[11], is applied. By satisfying (3), (6) and (8), the closedloop system will be decoupled in various DOFs. Therefore, stability of each DOF can be analyzed independently.

By some mathematical manipulations, the proposed system defined in (1)-(9) can be modeled as in Fig. 2 for each DOF; where $\tau_{1}$ and $\tau_{2}$ refer to communication delays from the patient to the therapist and vice versa; and:

$$
\begin{gathered}
\Xi_{1}(s)=\frac{Z_{\text {des,PFL }}(s)}{Z_{\vartheta, P F L}(s)+Z_{d e s, P F L}(s)+Z_{P F L}(s)} \\
\Xi_{2}(s)=\frac{1}{Z_{\vartheta, P I L}(s)+Z_{P I L}(s)} \\
\Xi_{3}(s)=-\frac{Z_{\varphi, T}(s)}{Z_{\vartheta, T}(s)+Z_{\varphi, T}(s)+Z_{T}(s)} \\
\Xi_{4}(s)=\frac{1}{Z_{\varphi, T}(s)} \\
\Xi_{5}(s)=\frac{1}{-Z_{d e s, P F L}(s)} \\
\Xi_{6}(s)=\left(\Xi_{1} \cdot \beta_{i}\right)-1 \\
Z_{(.)}(s)=M_{(.)} s^{2}+B_{(.)} s+K_{(.)} ; M_{(.)}, B_{(.)}, K_{(.)}>0
\end{gathered}
$$

In order to investigate the stability of the closed-loop system, a combination of the Small-Gain Theorem and the Circle Criterion is applied.

Theorem I [12]: The delayed feedback system given in Fig. 3 is Input-Output Stable (IOS) if:

$$
\begin{gathered}
u_{1} \in L_{\infty}, \quad u_{2} \in L_{\infty} \\
\zeta_{1} \in[0, \infty), \quad \zeta_{2} \in[0, \infty) \\
\zeta_{1} \cdot \zeta_{2} \leqslant 1
\end{gathered}
$$

where $\zeta_{1} \& \zeta_{2}$ stand for the IOS gain of sub-systems $\Sigma_{1}$ and $\Sigma_{2}$, respectively.

Definition I: The IOS gain of a system with the inputoutput relation $y(t)=\Sigma u(t)$, where $\Sigma$ is a mapping or operator that specifies $y$ in terms of $u$, is a nonnegative constant $\zeta$ such that:

$$
\sup _{t \geqslant 0}|y(t)| \leqslant \zeta \cdot \sup _{t \geqslant 0}|u(t)|+\varepsilon
$$

where $\varepsilon$ is a nonnegative constant bias term.

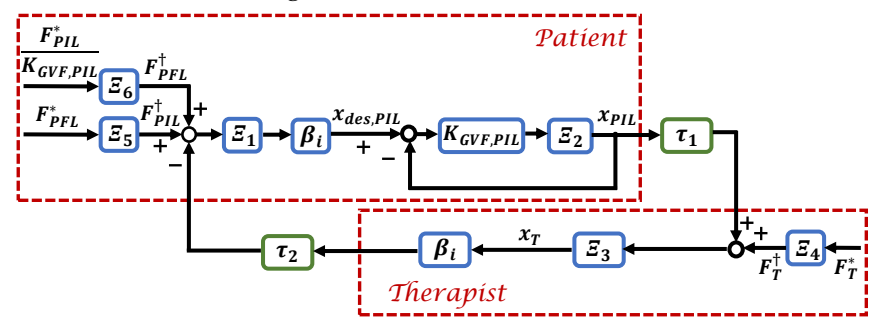

Fig. 2. The overall closed-loop system

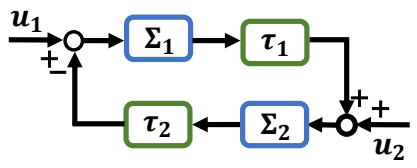

Fig. 3. Small-Gain Theorem 
Therefore, in order for the closed-loop system given in Fig. 2 to remain stable, the three small-gain conditions given in (17)-(19) should be met. Based on the first condition, it is required to have

$$
u_{1}=F_{P F L}^{\dagger}+F_{P I L}^{\dagger} \in L_{\infty} \quad, \quad u_{2}=F_{T}^{\dagger} \in L_{\infty}
$$

$F_{T}^{*}(t), F_{P F L}^{*}(t)$ and $F_{P I L}^{*}(t)$ refer to the exogenous forces applied by the therapist and the patient, which belong to the $L_{\infty}$ space [12], while $F_{T}^{\dagger}(t), F_{P F L}^{\dagger}(t)$ and $F_{P I L}^{\dagger}(t)$ indicate the outputs of the systems $\Xi_{4}(s), \Xi_{5}(s)$ and $\Xi_{6}(s)$ for inputs $F_{T}^{*}(t), F_{P F L}^{*}(t)$ and $\frac{F_{P I L}^{*}(t)}{K_{G V F, d e s}(t)}$, respectively. Having $0<\kappa_{\min }<$ $K_{G V F, P I L}$ from the previous section, the input $\frac{F_{P I L}^{*}(t)}{K_{G V F, d e s}(t)}$ is also bounded and belongs to the $L_{\infty}$ space. Considering the structure of systems $\Xi_{4}(s), \Xi_{5}(s)$ and $\Xi_{6}(s)$, which are stable and proper transfer functions belonging to the $L_{1}$ space, they map inputs in $L_{\infty}$ to outputs in $L_{\infty}$. Consequently, $F_{T}^{\dagger}(t)$, $F_{P F L}^{\dagger}(t)$ and $F_{P I L}^{\dagger}(t)$ belong to $L_{\infty}$, satisfying (17).

The next step in analyzing closed-loop stability is to check whether the IOS gains of the feedforward and the feedback paths in Fig. 2 satisfy the next two sets of conditions in (18) and (19). In order to calculate the IOS gain of the feedforward loop, first let us consider the local feedback loop in the feedforward path, from $x_{P I L}$ to $x_{d e s, P I L}$. In this feedback loop, $K_{G V F, P I L}$ is a time-varying parameter belonging to $\left[\kappa_{\min }, \kappa_{\max }\right]$, as defined in the previous section. This parameter refers to the stiffness of the GVF, to be adjusted adaptively. Without the need to go into details about how to design $K_{G V F, P I L}$, it can be assumed to belong to sector $(0, \rho]$ per the following definition:

Definition II [13]: A memoryless function $h:[0, \infty) \times$ $R^{P} \longrightarrow R^{P}$ is said to belong to the sector $(0, \rho]$ with $\rho=\rho^{T}>0$ if $h(t, u)^{T}[h(t, u)-\rho u] \leqslant 0$.

Stability of the local feedback loop from $x_{P I L}$ to $x_{d e s, P I L}$ can be analyzed using the Circle Criterion, as described next. Previously, Miandashti [14] used the Circle Criterion to study the stability of sampled-data bilateral teleoperation systems.

Theorem II [13]: The feedback connection of a linear dynamical system $G(s)$ and a nonlinear element $\xi$, as shown in Fig. 4, is stable if $\xi \in\left[\xi_{1}, \xi_{2}\right]$, with $\xi_{2}-\xi_{1}>0$, and $\left[I+\xi_{2} G(s)\right]\left[I+\xi_{1} G(s)\right]^{-1}$ is Strictly Positive Real (SPR).

It can be shown that the stability analysis for the feedback connection in Fig. 4, for $\xi=K_{G V F, P I L}(t)$, is similar to that of the feedback loop in Fig. 5, which in turn is similar to that for the local feedback loop in the feedforward path, from $x_{P I L}$ to $x_{d e s, P I L}$, in Fig. 2. Therefore, considering that $K_{G V F, P I L} \in\left[\kappa_{\min }, \kappa_{\max }\right]$ and based on Theorem II, the local feedback system, from $x_{P I L}$ to $x_{d e s, P I L}$, is stable if $\left[I+\kappa_{\max } \Xi_{2}(s)\right]\left[I+\kappa_{\min } \Xi_{2}(s)\right]^{-1}$ is SPR. According to the definition of an SPR transfer function [13], and considering the structure of $\Xi_{2}(s)$, which is a stable and strictly proper

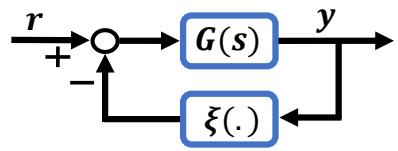

Fig. 4. Feedback connection used in Circle Criterion

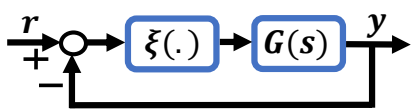

Fig. 5. Modified feedback connection used in Circle Criterion

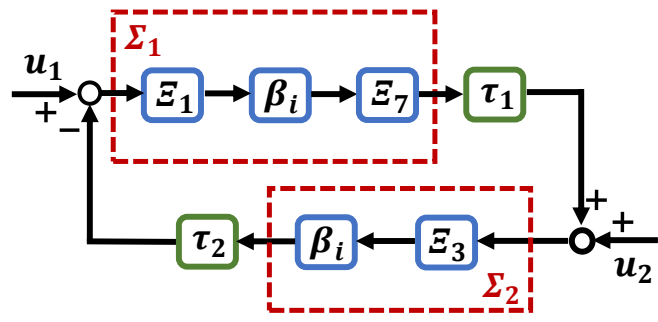

Fig. 6. The closed-loop system transformed based on the Circle Criterion

transfer function, $\left[I+\kappa_{\max } \Xi_{2}(s)\right]\left[I+\kappa_{\min } \Xi_{2}(s)\right]^{-1}$ is SPR if

$$
(1+\kappa)\left(K_{\Upsilon}+\kappa_{\min }\right)+B_{\Upsilon}^{2} \omega^{2}>(1+\kappa) M_{\Upsilon} \omega^{2}
$$

where $\kappa=\kappa_{\text {max }}-\kappa_{\text {min }}>0, M_{\Upsilon}=M_{\vartheta, P I L}+M_{P I L}, B_{\Upsilon}=$ $B_{\vartheta, P I L}+B_{P I L}$ and $K_{\Upsilon}=K_{\vartheta, P I L}+K_{P I L}$. Therefore, by proper adjustment of local control parameters at the PIL side $\left(M_{\vartheta, P I L}, B_{\vartheta, P I L}\right.$ and $\left.K_{\vartheta, P I L}\right)$, stability of the local feedback loop from $x_{P I L}$ to $x_{d e s, P I L}$ can be guaranteed. Having the local feedback loop stable, it can be shown that the loop has its highest input-output gain when $K_{G V F, P I L}$ is at its maximum level, i.e., $K_{G V F, P I L}=\kappa_{\max }$. Therefore, the IOS gain of the local feedback loop in the presence of time-varying $K_{G V F, P I L}$ will be equivalent to the IOS gain of the same loop when $K_{G V F, P I L}$ has been set to $\kappa_{\max }$. Therefore, we can continue the stability analysis of the overall closed-loop system by replacing the time-varying $K_{G V F, P I L}$ by its upper bound $\kappa_{\max }$, which represents the worst case. Consequently, Fig. 2 can be transformed to Fig. 6, where $\Xi_{7}(s)=\frac{\kappa_{\max } \cdot \Xi_{2}(s)}{1+\kappa_{\max } \cdot \Xi_{2}(s)}$. Comparing Fig. 6 with Fig. $3, \Sigma_{1}$ and $\Sigma_{1}$ can be written as

$$
\begin{gathered}
\Sigma_{1}(s)=\Xi_{1}(s) \cdot \beta_{i} \cdot \Xi_{7}(s)= \\
\frac{\beta_{i} \cdot \kappa_{\max }}{Z_{\vartheta, P I L}(s)+Z_{P I L}(s)+\kappa_{\max }} \cdot \frac{Z_{\text {des }, P F L}(s)}{Z_{\vartheta, P F L}(s)+Z_{\text {des }, P F L}(s)+Z_{P F L}(s)} \\
\Sigma_{2}(s)=\beta_{i} \cdot \Xi_{3}(s)=-\frac{\beta_{i} \cdot Z_{\varphi, T}(s)}{Z_{\vartheta, T}(s)+Z_{\varphi, T}(s)+Z_{T}(s)}
\end{gathered}
$$

The next step is to investigate condition given in (18), i.e., to have the IOS gains of $\Sigma_{1}(s)$ and $\Sigma_{2}(s)$ belong to $[0, \infty)$. Since $\Sigma_{1}(s)$ and $\Sigma_{2}(s)$ indicate transfer functions representing two LTI systems, the IOS gain is equal to the $L_{1}$ norm of the two systems; $L_{1}$ norm of transfer function $\Sigma(s)$ is defined according to the formula $\|\Sigma(s)\|_{L_{1}}=\int_{0}^{+\infty}|\sigma(\tau)| d \tau, \sigma(t)=$ $L^{-1}[\Sigma(s)]$. Therefore, (18) is equivalent to $\Sigma_{1}(s) \in L_{1}$ and $\Sigma_{2}(s) \in L_{1}$. Considering the structure of $\Sigma_{1}(s)$ and $\Sigma_{2}(s)$, which are stable and proper transfer functions, and knowing that $\beta_{i}$ and $\kappa_{\max }$ are bounded parameters, both $\Sigma_{1}(s)$ and $\Sigma_{2}(s)$ belong to $L_{1}$.

The last condition given in (19) necessitates

$$
\begin{gathered}
\left|\frac{\beta_{i} \cdot \kappa_{\max }}{Z_{\vartheta, P I L}(s)+Z_{P I L}(s)+\kappa_{\max }} \cdot \frac{Z_{d e s, P F L}(s)}{Z_{\vartheta, P F L}(s)+Z_{d e s, P F L}(s)+Z_{P F L}(s)}\right|_{L_{1}} \\
\left|-\frac{\beta_{i} \cdot Z_{\varphi, T}(s)}{Z_{\vartheta, T}(s)+Z_{\varphi, T}(s)+Z_{T}(s)}\right|_{L_{1}} \leq 1
\end{gathered} .
$$

which with some manipulations can be transformed into three 
conservative conditions, as follows:

$$
\begin{gathered}
\left|\kappa_{\max }\right| \leq\left|Z_{\vartheta, P I L}(s)+Z_{P I L}(s)+\kappa_{\max }\right| \\
\left|Z_{\text {des }, P F L}(s)\right| \leq\left|Z_{\vartheta, P F L}(s)+Z_{d e s, P F L}(s)+Z_{P F L}(s)\right| \\
\left|Z_{\varphi, T}(s)\right| \leq\left|Z_{\vartheta, T}(s)+Z_{\varphi, T}(s)+Z_{T}(s)\right|
\end{gathered}
$$

These three inequalities along with the one given in (21) represent the stability criteria for the closed-loop system in the presence of communication time delays between the patient and the therapist. As can be seen, the control parameters $M_{\vartheta, \Delta}, B_{\vartheta, \Delta}$ and $K_{\vartheta, \Delta} ; \Delta=P I L, P F L, T$ appear in all four conditions, through which the stability conditions can be satisfied.

Remark I: The proposed stability analysis platform can be possibly applied to general non-rehabilitation teleoperation applications, as well. The teleoperation framework itself can be considered as a new triple-user hierarchical leaderfollower system.

\section{EXPERIMENTS}

Two sets of experiments were conducted to evaluate the performance of the proposed framework. The experimental setup consists of two Quanser upper-extremity rehabilitation robots acting as the PIL and PFL robots; and one Quanser $\mathrm{HD}^{2}$ haptic device serving as the therapist's robot. The User Datagram Protocol (UDP) was used to transmit data between the master robots and the slave robot. The experimental setup is shown in Fig. 7.

The experiments were performed in two DOFs, along the sagittal-transverse plane. The mirroring between the PIL and the PFL was implemented along the sagittal axis. The experiments involved two scenarios, and two operators were asked to simulate behaviors of a typical patient and a typical therapist. The first scenario consisted of two phases to evaluate the mirroring effect between the PIL and the PFL, as well as the impact of the PFL as a medium on the therapist-commanded trajectory received at the PIL robot. The therapist was asked to generate and repeat a squared trajectory during both phases of the experiment. The patient was asked to consider the therapist-commanded trajectory as "comfortable" and "uncomfortable" in Phase I $(t=0-80 s)$ and Phase II $(t=80-160 s)$, respectively, and react accordingly. Therefore, she was supposed to intentionally alter the therapist-commanded trajectory by her PFL in the second phase, where the motions were defined as "uncomfortable". A time-varying profile was set for $K_{G V F, d e s}$, such that $\kappa_{\min }=$

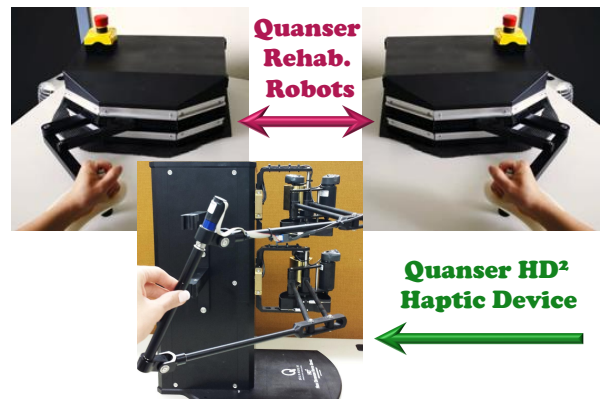

Fig. 7. Experimental Setup
350 and $\kappa_{\min }=400$. There was also $200 \mathrm{~ms}$ round-trip communication time delay introduced between the therapist's and the patient's robots.

The results are given in Figs. 8-10. Fig. 8 illustrates the 2D representation of the trajectories for the therapist, the PIL and the PFL. As can be seen, the therapist provided squared trajectories; the PFL followed the mirror-image of the Therapist-Commanded Trajectory (TCT), which in turn caused the PIL to follow the TCT in the same direction, as expected. In the second phase of the experiment, where the PFL was asked to resist the TCT due to the motions being considered as "uncomfortable" for the PIL, the amplitude of the PIL motion was also reduced through the PFL-mediated framework to avoid the painful/uncomfortable trajectory for the PIL. As can be seen, the framework also ensured the mirroring effect between the PIL and the PFL in both phases. Fig. 9 shows the same trajectory results in $1 \mathrm{D}$, along the mirroring axis with respect to time. Fig. 10 shows the force feedback provided to the therapist during the experiment. As can be seen, in the second phase, the therapist received considerable force on his hand informing him of the "discomfort" felt by the patient. This feature helps the therapist to be aware of and ensure the patient's safety.

The second experimental scenario investigated the effect of the time-varying virtual fixture gain $K_{G V F, \text { des }}$ on the PIL performance. For this purpose, a nonlinear time-varying profile was set for $K_{G V F, \text { des }}$, increasing from $\kappa_{\min }=1$ to $\kappa_{\max }=400$. The communication time delay between the patient's robots and the therapist's robot was $200 \mathrm{~ms}$ round-

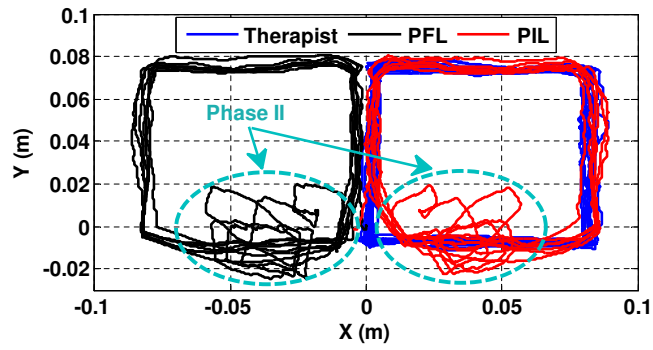

Fig. 8. Experimental Results - Scenario \#1: 2D plot of trajectories

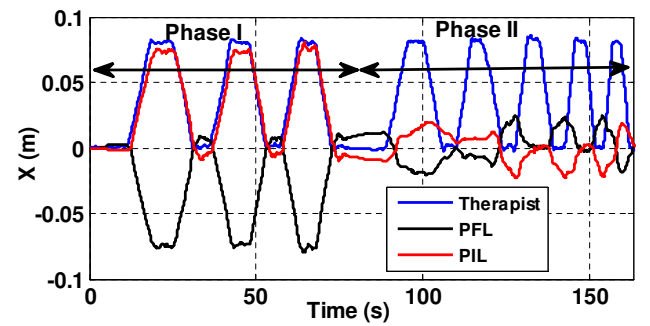

Fig. 9. Experimental Results - Scenario \#1: 1D plot of trajectories along the mirroring axis

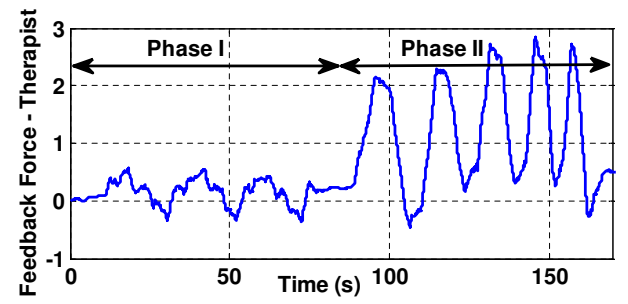

Fig. 10. Experimental Results - Scenario \#1: Haptic feedback provided to the therapist 

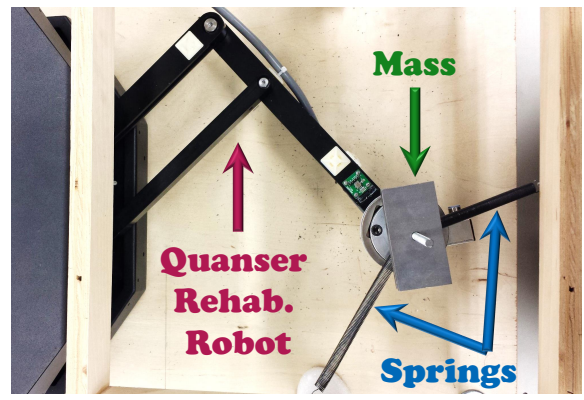

Fig. 11. The 2-DOF mass-spring array connected to the PIL robot

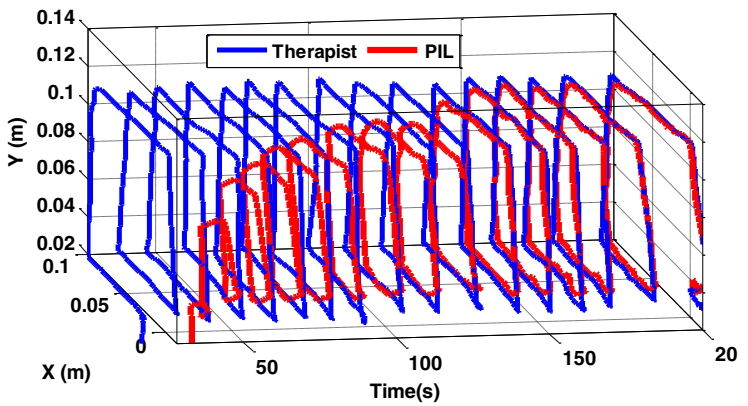

Fig. 12. Experimental Results - Scenario \#2: 2D trajectories with respect to time

trip. In order to simulate an impaired PIL, a 2-DOF massspring array was used to represent non-symmetric spasticity in a PIL. Spasticity, also referred to as an unusual stiffness, tightness, or pull of muscles, is a feature of altered skeletal muscle performance as a result of damage to the brain or the spinal-cord including that resulting from stroke.

For this purpose, the 2-DOF mass-spring array was connected to the PIL robot, as shown in Fig. 11, simulating an impaired PIL affected by spasticity. Similar to the first scenario, the therapist was asked to generate representative squared trajectories, while the PFL was asked to consider the TCT as comfortable, transferring the TCT to the PIL with no conditioning. Fig. 12 illustrates the 2-DOF timebased trajectory generated by the therapist and the trajectory followed by the simulated impaired PIL as a result of the time-varying GVF assistance force applied to the impaired PIL. As can be seen, at the beginning of the experiment, where $K_{G V F, d e s}$ was at its lowest value $K_{G V F, d e s}=\kappa_{\min }$, the GVF provided minimal assistance to the PIL, thus the PIL was not able to follow the therapist-commanded trajectory. By increasing $K_{G V F, \text { des }}$ during the experiment, the level of assistance provided to the PIL increased such that during the last 50s of the experiment, the impaired PIL fully tracked the desired TCT. Fig. 13 shows a 2D planar view of the same trajectories, where the smaller squares correspond to the lower levels of assistance by the GVF. As can be seen, at the beginning of the experiment, the simulated impaired PIL was not only unable to generate the desired amplitudes of the trajectory due to the low level of the GVF assistance, but also had an undesired rotational shift due to the asymmetry of the PIL. Towards the end of the experiment, increasing levels of the GVF assistance corrected for both amplitude and rotational-shift of the trajectories.

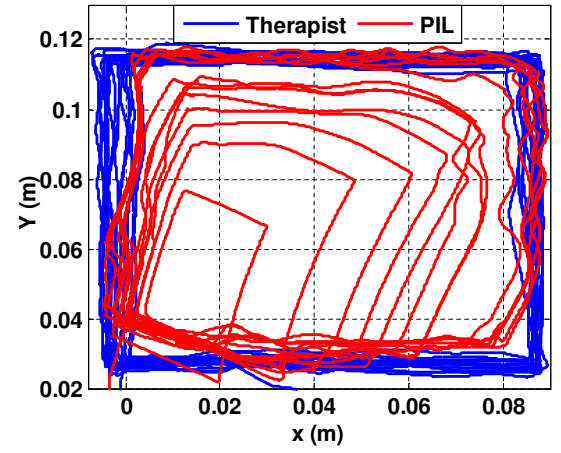

Fig. 13. Experimental Results - Scenario \#2: 2D plot of trajectories

\section{REFERENCES}

[1] S. Hesse, G. Schulte-Tigges, M. Konrad, A. Bardeleben, and C. Werner, "Robot-assisted arm trainer for the passive and active practice of bilateral forearm and wrist movements in hemiparetic subjects," Archives of physical medicine and rehabilitation, vol. 84, no. 6, pp. 915-920, 2003.

[2] C. G. Burgar, P. S. Lum, P. C. Shor, and H. M. Van der Loos, "Development of robots for rehabilitation therapy: the palo alto va/stanford experience," Journal of rehabilitation research and development, vol. 37, no. 6, pp. 663-674, 2000.

[3] J. H. Cauraugh and J. J. Summers, "Neural plasticity and bilateral movements: a rehabilitation approach for chronic stroke," Progress in neurobiology, vol. 75, no. 5, pp. 309-320, 2005.

[4] H. Kim, L. M. Miller, I. Fedulow, M. Simkins, G. M. Abrams, N. Byl, and J. Rosen, "Kinematic data analysis for post-stroke patients following bilateral versus unilateral rehabilitation with an upper limb wearable robotic system," IEEE Transactions on Neural Systems and Rehabilitation Engineering, vol. 21, no. 2, pp. 153-164, 2013.

[5] J. J. Summers, F. A. Kagerer, M. I. Garry, C. Y. Hiraga, A. Loftus, and J. H. Cauraugh, "Bilateral and unilateral movement training on upper limb function in chronic stroke patients: a TMS study," Journal of the neurological sciences, vol. 252, no. 1, pp. 76-82, 2007.

[6] P. Lum, C. G. Burgar, M. Van der Loos, P. Shor, M. Majmundar, and R. Yap, "The mime robotic system for upper-limb neuro-rehabilitation: results from a clinical trial in subacute stroke," in 9th International Conference on Rehabilitation Robotics, pp. 511-514, 2005.

[7] S. F. Atashzar, I. G. Polushin, and R. V. Patel, "Networked teleoperation with non-passive environment: Application to tele-rehabilitation," in IEEE/RSJ International Conference on Intelligent Robots and Systems, pp. 5125-5130, 2012.

[8] M. Shahbazi, S. F. Atashzar, and R. V. Patel, "A framework for supervised robotics-assisted mirror rehabilitation therapy," in 2014 IEEE/RSJ International Conference on Intelligent Robots and Systems, pp. 3567-3572, 2014.

[9] D. A. Lawrence, "Stability and transparency in bilateral teleoperation," IEEE Transactions on Robotics and Automation, vol. 9, no. 5, pp. 624637, 1993.

[10] M. Dyck and M. Tavakoli, "Measuring the dynamic impedance of the human arm without a force sensor," in 13th Int. Conf. Rehabilitation Robotics, 2013.

[11] M. Shahbazi, H. A. Talebi, S. F. Atashzar, F. Towhidkhah, R. V. Patel, and S. Shojaei, "A novel shared structure for dual user systems with unknown time-delay utilizing adaptive impedance control," in 2011 IEEE International Conference on Robotics and Automation, pp. 2124-2129, IEEE, 2011.

[12] I. Polushin, H. J. Marquez, A. Tayebi, and P. X. Liu, "A multichannel IOS small gain theorem for systems with multiple time-varying communication delays," IEEE Transactions on Automatic Control, vol. 54, no. 2, pp. 404-409, 2009.

[13] H. K. Khalil and J. Grizzle, Nonlinear systems, vol. 3. Prentice Hall, Upper Saddle River, 2002.

[14] N. Miandashti and M. Tavakoli, "Stability of sampled-data, delayed haptic interaction and teleoperation," in 2014 IEEE Haptics Symposium, pp. 215-220, IEEE, 2014. 